\title{
Mechanical fabrication of high-strength and redispersible wood nanofibers from unbleached groundwood pulp
}

\author{
Miikka Visanko (1) J Juho Antti Sirviö • Petteri Piltonen • Rafal Sliz • \\ Henrikki Liimatainen $\cdot$ Mirja Illikainen
}

Received: 24 March 2017 / Accepted: 8 July 2017 / Published online: 21 July 2017

(C) The Author(s) 2017. This article is an open access publication

\begin{abstract}
In the past, the direct production of lignincontaining nanofibers from wood materials has been very limited, and nanoscale fibers (nanocelluloses) have been mainly isolated from chemically delignified, bleached cellulose pulp. In this study, we have introduced a newly adapted, heat-intensified disc nanogrinding process for the enhanced nanofibrillation of wood nanofibers (WNF) with a high lignin content (27.4 wt\%). The WNF produced this way have many unique and intriguing properties in their naturally occurring form, for example, being able to be dispersed in ethanol and having ethanol solution viscosities higher than water solution viscosities. When WNF nanopapers were formed with ethanol, the properties of the nanofibers were recoverable without a notable decrease in the viscosity or mechanical strength after redispersing them in water. The preservation of lignin in the WNF was noticed as an increase in the water contact angles $\left(89^{\circ}\right)$, the rapid
\end{abstract}

Electronic supplementary material The online version of this article (doi:10.1007/s10570-017-1406-7) contains supplementary material, which is available to authorized users.

M. Visanko $(\bowtie) \cdot$ J. A. Sirviö · P. Piltonen ·

H. Liimatainen $\cdot$ M. Illikainen

Fibre and Particle Engineering Research Unit, University of Oulu, P.O. Box 4300, 90014 Oulu, Finland

e-mail: Miikka.Visanko@oulu.fi

R. Sliz

Optoelectronics and Measurement Techniques Unit, University of Oulu, P.O Box 4500, 90014 Oulu, Finland removal of water in the fabrication of the nanopapers, and the enhanced strength of the nanopapers when subjected to high pressure and heat. The nanopapers fabricated from the WNF were mechanically stable, having an elastic modulus of $6.2 \mathrm{GPa}$, a maximum stress of $103.4 \mathrm{MPa}$, and a maximum strain of $3.5 \%$. Throughout the study, characteristics of the WNF were compared to those of the delignified and bleached reference cellulose nanofibers. We envision that the exciting characteristics of the WNF and their lower cost of production compared to that of bleached cellulose nanofibers may offer new opportunities for nanocellulose and biocomposite research.

Keywords Nanogrinding · Nanofiber $\cdot$ Lignin · Viscosity $\cdot$ Nanopaper $\cdot$ Redispersion

\section{Introduction}

Lignocellulosic raw materials from plants (Bhatnagar and Sain 2005), trees (Herrick et al. 1983), and waste materials (Nair and Yan 2015a; Tarrés et al. 2017) have been studied to explore their suitability for nanocellulose production. The properties of fabricated nanofibers depend on the method of processing and the fiber origin. In plants, cellulose nanofibers tend to be more loosely bound, due to their structural and chemical composition (Valadez-Gonzalez et al. 1999), than in more organized wood structures having a high lignin content. Thus, the liberation of nanofibers from plant 
sources requires less chemical and mechanical processing, whereas wood-derived nanofibers (WNF) are mainly isolated solely from bleached, chemically delignified cellulose pulp. However, lignin-containing mechanical pulp fibers (e.g., thermomechanical pulp, or groundwood pulp; GWP) are attractive raw materials for nanofiber production at a price $50 \%$ lower (Arppe 2001) than that of chemical pulps and with a manufacturing yield of around 85-95\% (Sixta 2006). For this purpose, alternative processing methods are required to enable the breakage of the strong lignin matrix holds the wood fibers together. In addition, the inclusion of lignin components within the liberated nanofibers can result in a novel nanomaterial with chemical, mechanical, and surface properties different from traditionally fabricated cellulose nanofibers. This approach can also advance the discovery of novel applications without the need for the surface chemical functionalization of the nanofibers (Habibi 2014), leading to a reduction in environmental stress and costs in the production process. Above all, the conservation of lignin is of interest because it increases the hydrophobicity of the nanofibers, which could increase their use in multiple applications, for example, flotation (Laitinen et al. 2016), oil-water stabilization (Visanko et al. 2014a; Ojala et al. 2016), and biocomposites (Herzele et al. 2016; Winter et al. 2017).

The most effective methods for cellulose nanofiber production have so far been based on the use of chemical (Saito et al. 2007; Liimatainen et al. 2012, 2013b) or solvent (Selkälä et al. 2016) pretreatments for the surface functionalization of bleached pulp fibers. Chemical functionalization involves many drawbacks compared to the use of fibers in their native state, considering the high cost of chemicals, their toxicity, and the difficulties of both recycling (Kuutti et al. 2016) and regenerating them (Liimatainen et al. 2013a). Chemical treatments also tend to alter the properties of the resulting cellulose nanofibers, for example, by reducing the degree of polymerization (Lavoine et al. 2012) and drastically decreasing the thermal degradation threshold to around $200{ }^{\circ} \mathrm{C}$ (Fukuzumi et al. 2009; Eyholzer et al. 2010), which limits the ability of the fibers to be further processed (Oksman et al. 2016).

When cellulose nanofibers are dried, they can be formed into complex shapes and structures depending on the drying strategy used. High-strength (Sehaqui et al. 2012) transparent (Nogi et al. 2009) nanopapers with embedded functionalities (Sirviö et al. 2014a;
Morales-Narváez et al. 2015) and matrix properties (Sirviö et al. 2014b) can be obtained through simple vacuum filtration. The porosities of the fabricated nanopapers can be increased by replacing the water medium with solvents that are less polar (Sehaqui et al. 2011). Light-weight aerogels (Korhonen et al. 2011) with highly porous structures can be fabricated by freeze-drying, and the most convoluted drying methods of nanocelluloses under specific conditions enables the fabrication of highly organized and orientated aerogels (Donius et al. 2014).

The abundant hydroxyl groups and the high specific surface area of cellulose nanofibers increase their water uptake and the viscosity of aqueous nanofiber suspensions. This phenomenon is often experienced in the processing and fabrication of advanced materials such as nanopapers (Sehaqui et al. 2010). Consequently, the maximum operational consistency in cellulose nanofiber production is often limited to only $1-2 \%$, and the removal of water via filtration can be very timeconsuming. Moreover, in the commercialization of nanocelluloses, drying them is desired in many applications, but the redispersing of the nanofibers without hornification (Newman 2004) back to a solvent medium is still a challenge. Thus, the introduction of anionic charges through the use of additives (Lowys et al. 2001), the chemical modification of the fibers via carboxymethylation (Eyholzer et al. 2010; Butchosa and Zhou 2014), or the exploitation of nonwood plants with a high pectin content (Hiasa et al. 2016; Hietala et al. 2017) have been the most promising ways for recovering the properties of redispersed nanofibers. For nanofibers derived from wood, methods of drying and redispersing them in their native states in different media without the loss of any of their properties (Eyholzer et al. 2010), and without the use of any additives or chemicals, would be advantageous for the fabrication of green bioproducts.

So far, the direct production of nanofibers from nondelignified wood fibers has been limited to only a few methods. The direct grinding of the wood fibers has been attempted, but the production of fibers below micron-sized has not been possible (Spence et al. 2010; Hoeger et al. 2013). Currently, the most promising approaches to nanofibrillate wood fibers with a high lignin content originate from the partial delignification via ethanolysis (Winter et al. 2017) (17.3\% lignin) or via the use of fibers from the $\mathrm{SO}_{2}$-ethanol-water process (13.5\% lignin) (Morales et al. 2014; Rojo et al. 
2015). However, no study has yet addressed the heat response to and the softening of lignin at elevated temperatures, which could be one option for loosening the adhesive effect between the fibers, thus promoting the liberation of the nanofibers. Our goal was to produce, for the first time, WNF directly from nonchemically modified mechanical pulp without any delignification via combined high-temperature disc nanogrinding. The features of the resulting WNF were characterized, and a new, straightforward drying method for the efficient redispersion and property recovery of the WNF was investigated. The characteristics of the fabricated nanopapers based on the WNF and the impact of the drying temperature on the mechanical strength due to lignin melting have been demonstrated. A commercial bleached pulp and the cellulose nanofibers derived from it were used as reference materials.

\section{Experimental}

\section{Materials}

Unbleached spruce groundwood (GWP) in a neverdried form and a bleached, delignified sulfate spruce reference pulp (BP) in the form of dried pulp sheets were acquired from a pulp mill (Stora Enso, Veitsiluoto, Finland). Ethanol (96\%) for the solvent exchange of the nanofibers was purchased from VWR (Finland). Durapore polyvinylidene fluoride membranes for the fabrication of the nanopapers (pore size $0.65 \mu \mathrm{m}$ ) and track-etched Nuclepore membranes (pore size $0.20 \mu \mathrm{m}$ ) used for the preparation of the FE-SEM samples were acquired from Millipore (France). Deionized water was used throughout the study.

\section{Chemical composition of pulps}

Standard methods were used to analyze the compositions of the GWP and BP. The lignin content of the fibers was analyzed with a TAPPI T 222 om-02 (GWP $27.4 \mathrm{wt} \%$, BP $0.2 \mathrm{wt} \%$ ); the acetone-soluble extractives were analyzed with a TAPPI T 280 pm-99 (GWP $2.2 \mathrm{wt} \%$, BP $0.1 \mathrm{wt} \%$ ); and the hemicellulose and degraded cellulose portions were analyzed with a TAPPI T 212 om-02 (GWP 13.0 wt\%, BP 3.9 wt\%).
Nanogrinding of wood nanofibers and reference nanofibers

Disc nanogrinding of the GWP was performed with a 10-inch (c.25 cm) Masuko Super MassColloider (MKCA6-2 J CE; Masuko Sangyo, Japan) at $1500 \mathrm{rpm}$ at a high temperature. First, $60 \mathrm{~g}$ of the GWP was mixed with deionized water $(1.5 \mathrm{wt} \%)$ to a total weight of $4 \mathrm{~kg}$. With combined mixing and heating, an approximate temperature of $95{ }^{\circ} \mathrm{C}$ was reached prior to the grinding. The water cooler of the grinder was disconnected, and the grinder was preheated with boiling water to minimize heat loss. The heated fiber suspension was fed into the grinder in small portions to maintain the heat and was collected back into the heated cooking pans after each circulation. The heat of the fiber suspension was monitored with a digital thermometer and was found to vary from 90 to $95{ }^{\circ} \mathrm{C}$ during the passes through the discs. In total, the pulp passed once through to $0-$ and $-50-\mu \mathrm{m}$ disc clearances, and 15 times through the $100-\mu \mathrm{m}$ disc clearance. The reference sample was fabricated from the $\mathrm{BP}$ in the previously used conditions without heating and using the same grinding treatments as for the GWP. The reference sample, however, required a dilution below $1 \mathrm{wt} \%$ after the seventh pass through the $100-\mu \mathrm{m}$ disc clearance, as the viscosity of the sample was too high for the fiber slurry to flow through the discs.

Properties and morphology of the original pulps and nanofibers

The average (length-weighted) lengths and widths of the original pulp fibers were determined using a FiberLab image analyzer (Metso, Finland). The detailed visualization of the fibers was performed with a FE-SEM (Sigma HD VP, Germany) at an accelerating voltage of $3 \mathrm{kV}$. Prior to imaging, the fibers were disintegrated and diluted with water. From the dilutions, $5 \mathrm{ml}$ of the dispersed sample was filtered slowly with low pressure on top of a membrane (pore size $0.2 \mu \mathrm{m}$ ). The filtered samples, together with the membrane, were dipped into liquid nitrogen to freeze the fibers, and the specimens were immediately transferred to a freezer. The frozen samples were freeze-dried with a Coolsafe 55-15 Pro (ScanVac, Denmark) overnight. After freeze-drying, the 
specimens were fixed to sample holders, and the surfaces were sputter-coated with platinum $(\mathrm{Pt})$.

The morphological features of the fabricated nanofibers were also analyzed with a Tecnai G2 Spirit transmission electron microscope (TEM; FEI Europe, Eindhoven, the Netherlands). The samples were prepared by dilution with ultrapure water. A small droplet of the dilute solution was placed on top of a carbon-coated copper grid, which was first coated with polylysine by applying a small droplet of a $0.1 \%$ solution to the top of the grid. The excess polylysine was removed from the grid by touching the droplet with the corner of a piece of filter paper. A small droplet $(7 \mu \mathrm{l})$ of the diluted nanofiber suspension was then placed on the top of the grid and the excess was also removed from the grid with filter paper. Negative staining of the samples was performed by placing a droplet of uranyl acetate $(2 \% \mathrm{w} / \mathrm{v})$ on top of each specimen and the excess was removed with filter paper. The grids were dried at room temperature and analyzed at $100 \mathrm{kV}$ under standard conditions. Images were captured using a Quemesa CCD camera, and ImageJ image analysis software was used to analyze the nanofiber dimensions.

\section{Viscosity}

The rheological analysis of the nanofibers was conducted in water (W) and ethanol (E). For the viscosity measurements in ethanol, the nanofiberwater suspensions were gradually solvent-exchanged. The nanofibers were first dispersed into ethanol and mixed for $24 \mathrm{~h}$, during which time the ethanol was removed three times by vacuum filtration and replaced with fresh solvent. The viscosities were measured at consistencies of $0.5,1$, and $1.5 \mathrm{wt} \%$ for the WNF and at $0.3 \mathrm{wt} \%$ for the reference nanofibers. The measurements were conducted with a DV-II+Pro EXTRA viscometer (Brookfield, USA) at a temperature of $20{ }^{\circ} \mathrm{C}$ using a vane-shaped spindle (V-73) and rotational speeds of $10,20,50$, and $100 \mathrm{rpm}$.

\section{Preparation of nanopapers}

The WNF and reference nanofibers were used to prepare nanopapers for the detailed characterizations of the nanofibers. First, $0.33 \mathrm{~g}$ abs. of the nanofibers were diluted to $0.3 \mathrm{wt} \%$ (corresponding to nanopapers with grammage of $80 \mathrm{~g} / \mathrm{m}^{2}$ ) and were further mixed with a magnetic stirrer to ensure the formation of evenly dispersed suspensions. Vacuum filtration with a glass filter funnel $(7.2 \mathrm{~cm}$ diameter) covered with a polyvinylidene fluoride membrane (pore size $0.65 \mu \mathrm{m}$ ) was performed for the nanopaper fabrication. The filtration time for the manufacture of the nanopaper was recorded. After filtration, the moist nanopaper that was formed was peeled off the membrane and placed between two filter papers. The drying of the specimens was performed with a hot press (Fontune Press, the Netherlands) in two consecutive steps (Table 1). In step 1, the aim was to dry the sample using a low pressure of $25 \operatorname{bar}(F=10 \mathrm{kN})$ together with the filter papers. In step 2 , the dried nanopaper was placed between the hot metal plates without the filter papers and pressed at a high pressure of $740 \mathrm{bar}(F=300 \mathrm{kN})$ to remove any possible residual moisture and to fabricate more-compacted nanopapers. The labeling of the nanopapers produced from the WNF and the reference nanofibers from here on are based on the applied temperature used in the drying and pressing (Table 1). After pressing, the samples were stored at standard conditions of $23{ }^{\circ} \mathrm{C}$ and $50 \%$ relative humidity. The densities of the nanopapers were determined after preconditioning for at least $48 \mathrm{~h}$ in the standard environment. The weight and accurate volume (width $\times$ thickness) of each sample were measured, and the densities were calculated.

\section{Crystallinity}

The crystallinities of the original GWP, BP, and the nanofibers were measured from sheets (pulps) or nanopapers (WNF 150 and REF 150) using wideangle $\mathrm{x}$-ray diffraction. The measurements were conducted on a Rigaku SmartLab x-ray diffractometer equipped with a $\mathrm{Co} \mathrm{K} \alpha$ radiation source $(k=$ $0.1789 \mathrm{~nm}$ ). The samples were mounted on a solid circular holder, and a high-speed $1 \mathrm{D}$ detector $(\mathrm{D} / \mathrm{Tex}$

Table 1 Time and temperature used in the pressing steps for the fabrication of nanopapers from WNF and reference nanofibers

\begin{tabular}{lll}
\hline Nanopaper & Step 1 (25 bar) & Step 2 (740 bar) \\
\hline WNF 75 & $24 \mathrm{~h}, 75^{\circ} \mathrm{C}$ & $3 \mathrm{~min}, 75^{\circ} \mathrm{C}$ \\
WNF 100 & $60 \mathrm{~min}, 100^{\circ} \mathrm{C}$ & $3 \mathrm{~min}, 100^{\circ} \mathrm{C}$ \\
WNF 150 & $3 \mathrm{~min}, 150{ }^{\circ} \mathrm{C}$ & $3 \mathrm{~min}, 150{ }^{\circ} \mathrm{C}$ \\
REF 150 & $3 \mathrm{~min}, 150{ }^{\circ} \mathrm{C}$ & $3 \mathrm{~min}, 150{ }^{\circ} \mathrm{C}$ \\
\hline
\end{tabular}


Ultra 250; Rikagu) was set to collect the data. The scanning range was from $10^{\circ}$ to $50^{\circ} 2 \theta$ with a step width of $0.02^{\circ}$ and a scanning speed of $1{ }^{\circ} \mathrm{min}^{-1}$. The degree of crystallinity in terms of the crystallinity index (CrI, Eq. 1) was calculated based on the reflected intensity data, following the method of Segal et al. (1959):

$\mathrm{CrI}[\%]=\left(I_{200}-I_{\mathrm{am}}\right) / I_{200} \times 100 \%$

where $I_{200}$ is the peak intensity corresponding to both the amorphous and crystalline fractions of cellulose $I$, and $I_{\mathrm{am}}$ is the peak intensity of the amorphous fraction.

\section{Mechanical properties of nanopapers}

The tensile tests were performed with a universal material testing machine (Instron 5544, USA) equipped with a 100-N load cell. The nanopapers were cut into 5-mm-wide strips, and their thicknesses were measured using a precision thickness gauge (Hanatek FT3, UK). Three different locations along the gauge length were measured to calculate the average thickness. For the tensile testing, a 40-mm gauge length was set under a strain rate of $4 \mathrm{~mm} / \mathrm{min}$, and five strips were measured for each sample. The tests were conducted at a relative humidity of $50 \%$ at a temperature of $23{ }^{\circ} \mathrm{C}$ using a preload of $\sim 0.1 \mathrm{~N}$. Prior to testing, the specimens were conditioned for at least 1 day in the same environment. The elastic modulus was calculated from the initial linear part of the stressstrain curve, and the maximum stress and strain at break were determined from specimen breakage.

Surface properties and morphology of nanopapers

The nanopaper surfaces and cross-sectional structures were imaged with an FE-SEM (Zeiss ULTRA Plus, Germany) at an accelerating voltage of $5 \mathrm{kV}$. The cross-sectional images of the nanopapers were taken from the specimens' fractured surfaces after tensile testing. Prior to imaging, the specimens were sputtercoated with Pt.

A static sessile-drop contact angle measurement method was used to measure the water contact angles of the nanopapers fabricated from WNF 150 and REF 150. Milli-Q water was used as a probe liquid at room temperature with a Krüss DSA100 system (Germany). The instrument was equipped with a high-speed camera (1000 fps) and analysis software. The contact angle was determined immediately after the formation of a drop on the nanopaper surface. The contact angles were extracted by the height-width method, where a rectangle enclosed by a contour line is regarded as being the segment of a circle. As a result, contact angles can be calculated from the height-width relationship of the enclosing rectangle. For each sample, five droplets at different locations were studied, the results were averaged, and the standard deviations were calculated.

Thermogravimetric analysis

TGA was performed on the original pulps (GWP and $\mathrm{BP}$ ), ground reference nanofibers, and WNF. The samples were frozen in a freezer and then freeze-dried prior to the analysis. The measurements were taken with an STA 409 PC (Netsch, Germany) under a nitrogen atmosphere with a flow speed of $60 \mathrm{ml} / \mathrm{min}$. The specimens were placed on a sample carrier and heated from room temperature to $700{ }^{\circ} \mathrm{C}$ at a heating speed of $10{ }^{\circ} \mathrm{C} / \mathrm{min}$.

\section{Redispersion of dried WNF and reference nanofibers}

The ability to redisperse dried nanofibers into water was investigated by freely-dried nanopapers from the WNF and reference nanofibers. Two routes for the fabrication of nanopapers were investigated by using nanofibers dispersed in water or ethanol. The solvent exchange to ethanol was performed as described earlier (see "Viscosity"). For the preparation of nanopapers, $1 \mathrm{~g}$ abs. of nanofibers was diluted to a $0.3-w t \%$ consistency (with water or ethanol) and were mixed with a magnetic stirrer. The suspensions were vacuum-filtered on top of a membrane $(0.65-\mu \mathrm{m}$ pores), and after nanopaper formation, the samples were allowed to dry freely overnight at room temperature. The dried nanopapers were redispersed back into the water by cracking them into smaller pieces and mixing them with water to obtain suspensions with a $1.5-w t \%$ consistency. The mixing was performed with a hot-plate magnetic stirrer, and the temperature of the suspension was adjusted to $50{ }^{\circ} \mathrm{C}$. After 30 min of mixing, the remaining large flocs were disintegrated with an UltraTurrax mixer (IKA T25, Germany) at $12,000 \mathrm{rpm}$ for $2 \mathrm{~min}$. The magnetic stirring was continued under heat for another $90 \mathrm{~min}$ 
after the UltraTurrax treatment. The redispersed samples were labeled according to the medium used in the fabrication of the nanopapers: redispersed from nanopaper dried in water (WNF RW and REF RW) or redispersed from nanopaper dried in ethanol (WNF RE and REF RE).

The viscosities of redispersed nanofiber suspensions were measured at consistencies of $1.5 \mathrm{wt} \%$ for the WNF and $0.3 \mathrm{wt} \%$ for the reference nanofibers. The measurements were conducted according to the same patterns described earlier (see "Viscosity"). Finally, nanopapers of $80 \mathrm{~g} / \mathrm{m}^{2}$ were prepared from the redispersed samples according to the same procedures stated earlier (see "Preparation of nanopapers"). However, the pressing was conducted at a $150{ }^{\circ} \mathrm{C}$ (steps 1 and 2; see Table 1) for both the WNF and reference nanopapers. The pressed and dried nanopapers were pre-conditioned at a relative humidity of $50 \%$ and a temperature of $23{ }^{\circ} \mathrm{C}$ for $24 \mathrm{~h}$ prior to measuring their mechanical properties, as previously described (see "Mechanical properties of nanopapers").

\section{Results and discussion}

Nanogrinding of fiber specimens

A newly adapted, heat-intensified disc nanogrinding process was tested for the direct preparation of WNF with a high lignin content (27.4 wt $\%$ ) from unbleached GWP. The relationship between high-temperature lignin softening and the ease of liberation of more intact wood fibers has been well known and used for decades in the production of refined mechanical and ground pulp (McDonald et al. 2004). Thermoplasticity studies of wood have shown different softening temperatures for lignin depending on the conditions, and, at a water saturation state of $20 \%$ or higher, it has been proposed that the softening takes place from 72 to $128{ }^{\circ} \mathrm{C}$ (Goring 1963). Our aim was to maintain the temperature of the pulp at approximately $90-95{ }^{\circ} \mathrm{C}$ over the course of the nanogrinding, during which the lignin was expected to soften, thus enabling the successful liberation of nanofibers directly from the wood fibers. We used an open system operating in a normal atmosphere with a temperature close to the boiling point of water, causing the water to evaporate. Consequently, the consistency of the GWP suspension rose almost threefold from the initial 1.5-4.3 wt\% during the treatment. Yet, this increase did not cause any difficulties in processing the sample at any stage, making this technique a promising one for markedly increasing the production capacity when compared to the use of bleached cellulose fibers (Hu et al. 2015). The low operating consistency of BP was evident, and a dilution to below $1 \mathrm{wt} \%$ had to be made in the intermediate stage of processing to reduce the strong gelling and the increase in the viscosity of the sample.

\section{Morphology of nanofibers}

The original fibers from the GWP and the reference pulp had length-weighted widths of 26.1 and $26.8 \mu \mathrm{m}$ as well as lengths of 0.78 and $1.76 \mathrm{~mm}$, respectively (Fig. S1). The shorter fiber length of the GWP was due to the initial mechanical pulping, which resulted in fiber cutting. After the high-temperature disc nanogrinding, we were able to obtain WNF with a very high lignin content directly from the GWP without any chemical modifications. As far as we know, the nanofibers had the highest lignin content reported thus far.

According to images captured by a transmission electron microscope (TEM), the obtained WNF had a heterogenous size distribution, but the dimensions were well within the nanoscale (Fig. 1). We classified the nanofibers seen in the images into two fractions based on their sizes. In the first fraction $(5-50 \mathrm{~nm})$, the nanofibers aggregated with the majority of their typical widths varying from 10 to $35 \mathrm{~nm}$ (Fig. 1a, b, $\mathrm{d}, \mathrm{e})$, and the nanofibers were found to be abundant close to concentration gradients of highly negatively stained areas. Individual nanofibers (lateral dimension $\sim 5 \mathrm{~nm}$ ) were also occasionally found. A typical example of an area covered by a WNF network is shown in Fig. 1e. The second fraction was classified as nanofiber bundles $(50-300 \mathrm{~nm})$, which appeared in random areas in the imaged grids (Fig. 1c). These bundles were often partially nanofibrillated and were seen to unravel from their ends into multiple smaller nanofiber aggregates. The darker areas in the images were caused by the negative staining of uranyl acetate, and the white dots or irregular stains in the background were caused by polylysine. The surfaces of the WNF seemed to be receptive to polylysine, and these places 


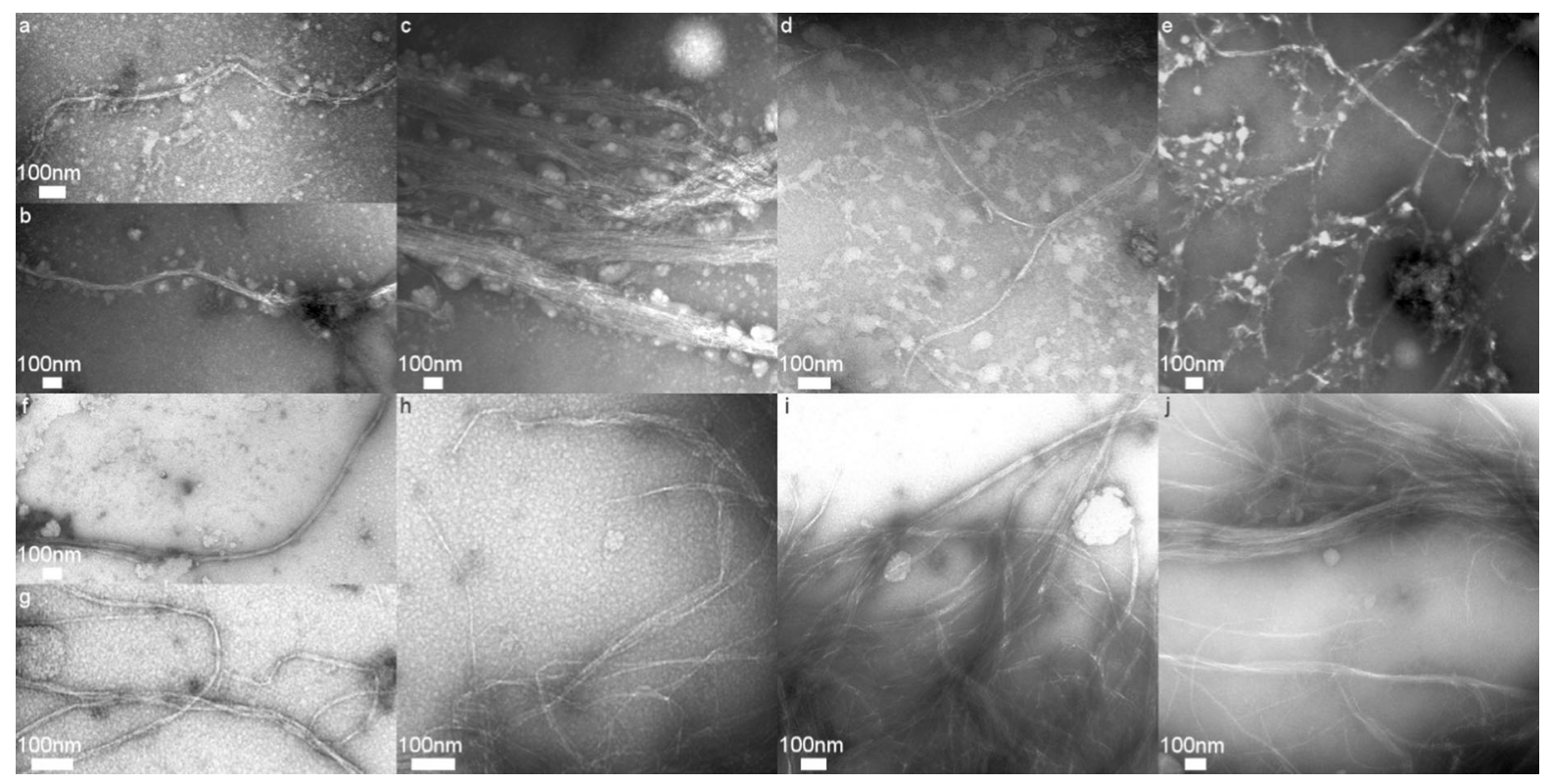

Fig. 1 TEM images of WNF (a-e) and reference nanofibers $(\mathbf{f}-\mathbf{j})$ at different magnification levels

could be seen as white dots on the outside parts of the nanofibers (Fig. 1c).

Bleached and delignified cellulose fibers have been reported to disintegrate into more evenly sized nanofibers when exposed to strong shear and tearing forces in a disc grinder (Abe et al. 2007). Here, a more homogeneous size distribution of nanofibers was obtained for the reference pulp than for the GWP. The lignin-free cellulose fibers of the reference pulp were likely more swollen, loosely bound, and prone to nanofibrillation than the lignin-containing GWP. The majority of the imaged reference nanofibers had a typical width of around 7-20 nm. No larger nanofiber bundles (50-300 nm) were found in the TEM images. Examples of the typical widths of the nanofibers are given in the supporting information (Fig. S2; Table S1).

The crystallinity indexes (CrI) calculated from the $\mathrm{x}$-ray diffraction diffractograms (Fig. S3) were 78.1 and $57.3 \%$ for the original pulps (BP and GWP), respectively, and 71.6 and $58.3 \%$ for the reference nanofibers and the WNF, respectively. The GWP underwent initial mechanical pulping, which caused the degradation of the crystalline domains of the fibers (Clark and Terford 1955), whereas the BP was obtained directly from chemical pulping. The differences in the initial CrIs between the specimens thus originated from the pretreatment of the fibers. After the disc nanogrinding, the BP without any mechanical pretreatment (with a more intact crystalline structure) was susceptible to a larger drop in the crystallinity as the result of a reduction in the chain length and the crystal size. For the GWP, no decrease was observed in the $\mathrm{CrI}$ after the heat-intensified disc nanogrinding.

Rheology of nanofiber suspensions

The rotational viscosities of the WNF and reference nanofibers were measured at different consistencies in water and ethanol suspensions. The WNF water suspensions had a remarkably lower viscosity than those of the reference nanofiber suspensions, and a consistency approximately five-times thicker (1.5 vs. $0.3 \mathrm{wt} \%$ ) was required to obtain a similar torque resistance (Fig. 2). The lower viscosity of the WNF was probably caused by the occurrence of coarser nanofibers, the stiffness of the WNF, a lower water adsorption, and a reduced polarity due the presence of lignin. The confined gelling of the WNF confirmed the prospect of the elevated consistency processing observed in the grinding of the GWP. Another interesting property seen in the WNF was their ability to disperse and to form stable suspensions when solvent-exchanged to ethanol. Interestingly, the surface chemical composition of the WNF was more compatible with less-polar ethanol (WNF E), and the 


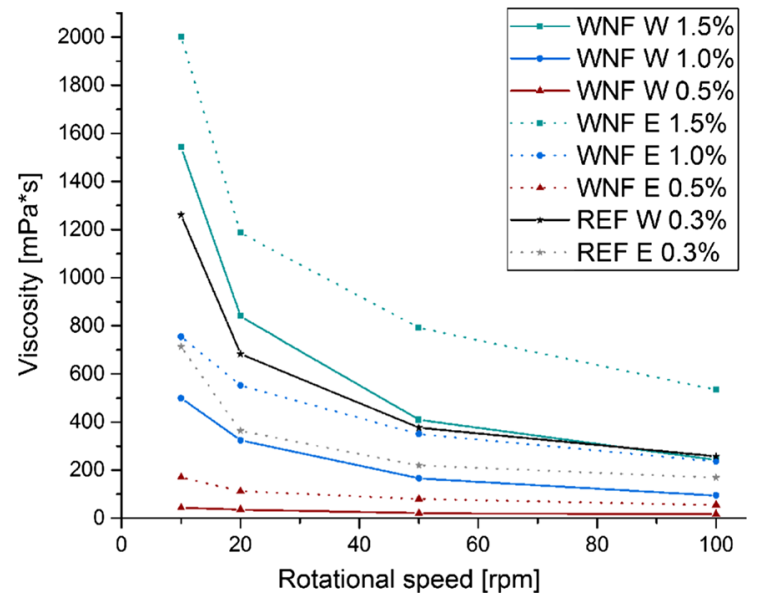

Fig. 2 Viscosity of the wood nonfibers $(W N F)$ and the reference nanofibers $(R E F)$ in water $(W)$ or ethanol $(E)$ at different consistencies $(0.3-1.5 \%)$

viscosities of all the studied consistencies were higher for ethanol than for water (WNF W). The reference nanofibers, in turn, had a reduced viscosity in ethanol (REF E). Typical shear-thinning behavior was seen in both the reference nanofibers and the WNF, with higher consistencies of 1.5 and $1.0 \mathrm{wt} \%$, respectively, in both water and ethanol. The ability of the WNF to be easily dispersed in a nonaqueous solvent may be beneficial in many applications. For example, in nanocomposite processing, less-polar solvents are advantageous for achieving a homogenous mixture on hydrophobic matrices and improved compatibility with a greater reinforcing effect.

\section{Nanopaper properties}

Nanopaper fabrication was performed by filtering the aqueous water-nanofiber suspension and subsequently pressing and drying. Despite the advantages of nanopapers, the lengthy filtration time required for film formation has been a troublesome bottleneck in the fabrication process for nanopapers. Our WNF showed great potential for the rapid preparation of nanopapers, with an average filtration time of only $3.5 \mathrm{~min}$. The reference nanofibers, in contrast, required $44 \mathrm{~min}$ for the removal of the residual water prior to qualifying for the drying steps. The hydroxyl group-rich surfaces of cellulose nanofibers have a tendency toward high water adsorption and swelling, and thus strong gel formation, which was also apparent in the viscosity measurements. The WNF with a high lignin content possessed low water adsorption capabilities, and the stiffer nanofibers formed a lessresistant, porous filtration layer that enabled a higher water flux (Rojo et al. 2015).

During the fabrication of the nanopapers, different pressing times and temperatures were applied to study how their properties developed, with particular attention to the role of the possible thermal softening of lignin. Field-emission scanning electron microscope (FE-SEM) surface images taken from WNF 75 indicated a higher porosity at the pressing temperature of $75{ }^{\circ} \mathrm{C}$ (with more openings in the surface noted; Fig. 3a), which was reduced when the pressing temperature was increased to $100{ }^{\circ} \mathrm{C}$ (Fig. 3b). With a $150{ }^{\circ} \mathrm{C}$ pressing temperature, visible pores started to disappear, and more distinct flat areas were observed in the nanopaper surface (Fig. 3c). The flatter areas were assumed to have formed due to lignin softening and the filling of small voids at an elevated temperature. For REF 150, some random larger nanofibers were observed, but, in general, the overall surface structure was smooth with no porosity (Fig. 3d), and the partly flattened areas seen in WNF 150 were not present.

The tensile properties of the nanopapers from WNF were also affected by the applied drying parameters. The tensile strength, strain-at-break, and elastic modulus improved with increased drying temperatures (Fig. 4). The increment especially in the stiffness and strength for WNF 100 and WNF 150 compared to WNF 75 supports the earlier findings that melting of lignin at an elevated temperature leads to the formation of a more compact structure (Rojo et al. 2015). This was also noted in the increase in densities among the WNF specimens (Table 2). Generally, the mechanical properties of the reference nanopaper (REF 150) were superior to those of the WNF (WNF 75-150) but similar to those of previously reported cellulose nanofiber-based nanopaper (Nair et al. 2014). This behavior originates from the differences in the nanofiber surface properties, the narrower size distribution, and the finer width obtained from the reference nanofibers. The more homogenous composition and flexibility of the reference nanofibers can be expected to result in abundant contact points, with the strength enhanced by strong hydrogen bonding within the nanopaper network. The high lignin content of the WNF may, in turn, decrease the adhesion and strength of bonds between the nanofibers. In addition, the 


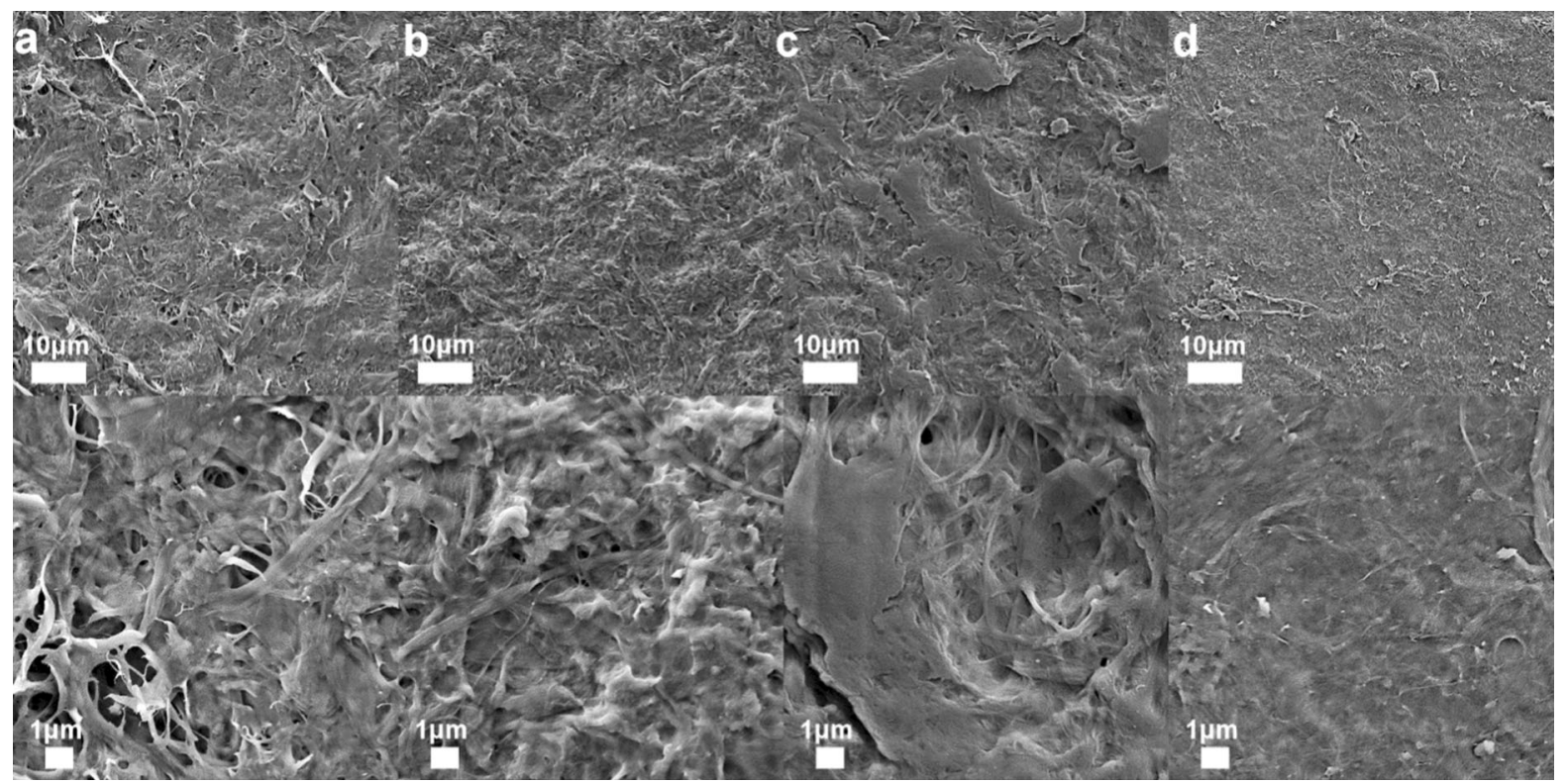

Fig. 3 FE-SEM images of the nanopaper surfaces for WNF 75 (a), WNF 100 (b), WNF 150 (c), REF 150 (d), and their corresponding magnifications

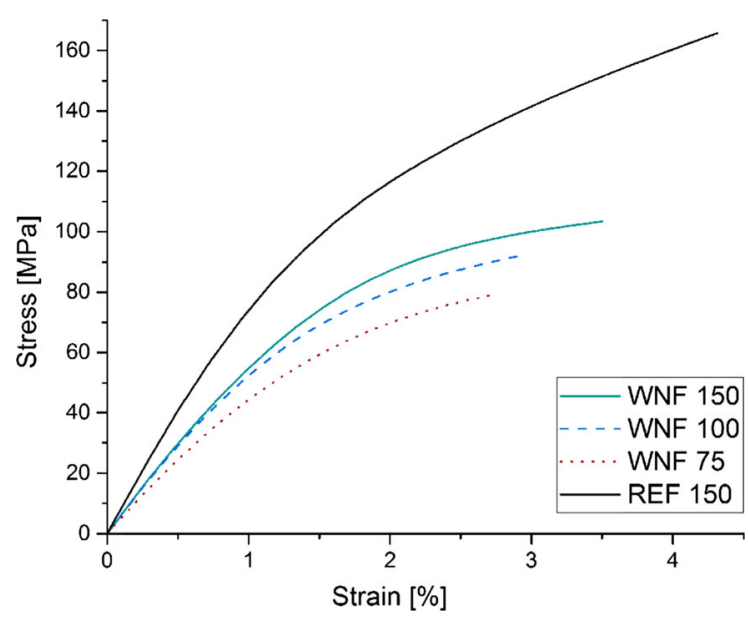

Fig. 4 Averaged stress-strain curves for WNF and REF nanopapers mechanical properties of a single WNF are expected to be weaker due to the incorporation of lignin, a phenomenon that has been demonstrated with wood fibers (Zhang et al. 2013). The density of REF 150 was also higher than that of the WNF nanopapers, and, overall, the strength of the specimens was seen to positively improve with the higher temperature used in the pressing. For the later analysis of the nanopapers, fabrication at $150{ }^{\circ} \mathrm{C}$ was selected for both WNF and $\mathrm{REF}$, as that temperature provided the most promising properties. Also, in terms of practicality, drying at $150{ }^{\circ} \mathrm{C}$ was the most feasible option, as it required the least amount of time for the evaporation of any residual water.

The surface properties of WNF 150 and REF 150 were further analyzed to understand the differences in

Table 2 Mechanical properties of nanopapers and their densities

\begin{tabular}{lllcr}
\hline Sample & Elastic modulus $(\mathrm{GPa})$ & Strain at break $(\%)$ & Maximum tensile strength $(\mathrm{MPa})$ & Density $\left(\mathrm{kg} / \mathrm{m}^{3}\right)$ \\
\hline WNF 75 & $5.1 \pm 0.5$ & $2.7 \pm 0.4$ & $79.0 \pm 7.1$ & 1210 \\
WNF 100 & $6.0 \pm 0.4$ & $2.9 \pm 0.7$ & $91.8 \pm 5.3$ & 1260 \\
WNF 150 & $6.2 \pm 0.3$ & $3.5 \pm 0.3$ & $103.4 \pm 2.6$ & 1300 \\
REF 150 & $8.3 \pm 0.7$ & $4.3 \pm 0.6$ & $165.8 \pm 9.4$ & 1440 \\
\hline
\end{tabular}


the nanopaper features. The static water-contact angles (WCA) measured from the nanopaper surfaces were $88.6^{\circ} \pm 1.6^{\circ}$ and $70.3^{\circ} \pm 1.2^{\circ}$ for WNF 150 and REF 150, respectively. The high lignin content of the WNF was seen in the more water-repellent interface of the nanopapers compared to REF 150. Lignin has a variety of functional groups on its surface, making it more hydrophobic (Laurichesse and Avérous 2014) than hydroxyl group-rich cellulose. The WCAs were higher than those reported for the nanopapers fabricated from WNF $\left(77.8^{\circ}\right)$ with a 13.5 -wt $\%$ residual lignin (Rojo et al. 2015), but in reasonable agreement with our sample that contained twice as much lignin. Rojo et al. (2015) also saw an increase in WCAs with rising lignin content, but stated that their initial chemical pulping of the fibers modified the residual lignin to be more hydrophilic. For REF 150, the results were similar to those reported for nanopaper fabricated from softwood cellulose nanofibers $\left(\sim 66^{\circ}\right.$; Kisonen et al. 2015).

WNF 150 had a brownish-yellow coloring due to the presence of unremoved wood components; in comparison, REF 150 consisted mainly of bleached cellulose and appeared white with some translucent areas (Fig. 5). The cross-sections from the nanopapers showed structural differences between the specimens. REF 150 showed a layered structure that is typical for nanopaper (Li et al. 2017), while WNF 150 had layers with grooves and a less-aligned horizontal positioning (high-magnification images of the cross-sections can be seen in Fig. S4). The inconstant, irregular structure stems from the broader size distribution of the WNF in contrast to the reference nanofibers. Also, the fractured interiors of the nanopapers were seen to break in a smooth way in REF 150, while WNF 150 had a more uneven surface with some outward-projecting filaments.

\section{Thermogravimetric analysis (TGA)}

Thermal stability in the emerging temperature profile was affected by the compositional and structural differences between the specimens. The samples from the BP contained mainly cellulose, whereas wood fibers can be classified as nature's own complex biocomposite that includes multiple other components (lignin, hemicellulose, extractives, etc.), which decompose at different temperatures (Morán et al. 2008) and can intensify the degradation process

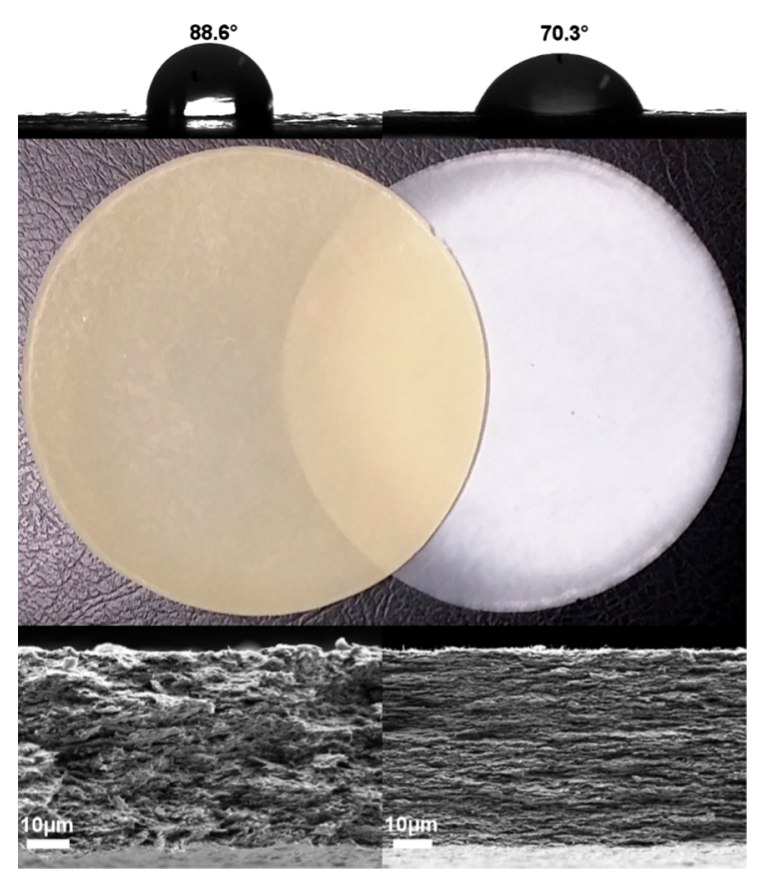

Fig. 5 Water contact angle, visual appearance, and crosssectional structure of nanopapers from WNF 150 (left) and REF 150 (right)

(Poletto et al. 2012). This was observed in the lower onset temperature of the GWP and the larger final char content (related to high lignin content; Fig. 6a), which is known to be more difficult to decompose at a slower rate over a broader temperature range than cellulose (Gani and Naruse 2007; Yang et al. 2007). In addition, the BP had a higher crystallinity, which improved the thermal stability of the fibers (Kim et al. 2010).

The minor initial weight-loss peak observed in the derivative thermogravimetric curves (Fig. 6b) around $90{ }^{\circ} \mathrm{C}$ was associated with the evaporation of the residual humidity in the samples (Chen et al. 2011). The reduction in the fiber size and the increase in the surface area led to a more rapid mass loss, a lower onset temperature, and an earlier degradation of the reference nanofibers in comparison with its micronsized fibers (Quiévy et al. 2010; Sirviö et al. 2015). With the WNF, this effect was more restrained, and only a minor shift to a lower temperature at the degradation peak was noted. The preservation of the thermal properties of the nanofibers is desirable for processing the materials but was difficult to maintain in previous studies with chemically modified cellulose nanofibers (Fukuzumi et al. 2009; Eyholzer et al. 2010). Here, it is important to note that our WNF were 


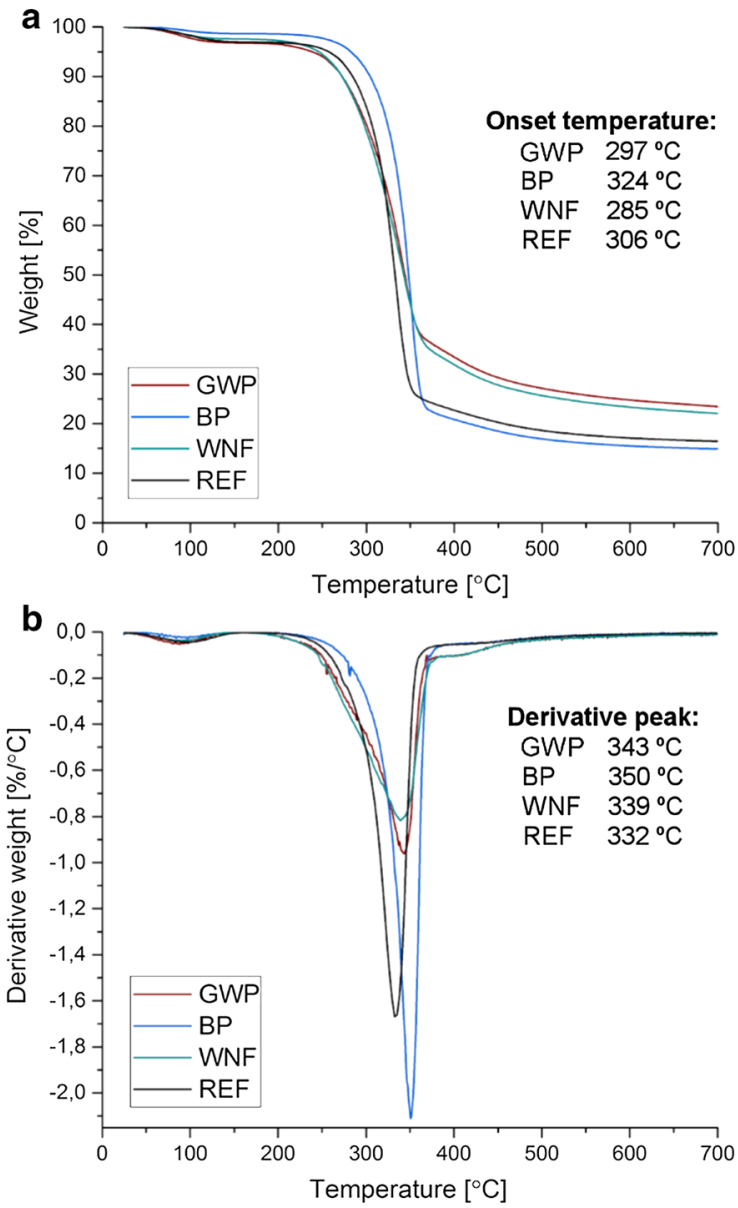

Fig. 6 TGA (a) and DTG (b) measured in a nitrogen atmosphere

able to retain an onset temperature and degradation rate close to those of the original fibers, with only a minor decline. According to a recent study (Nair and Yan 2015b), an initial 1\% NaOH treatment of fibers with a high lignin content could further increase the thermal stability of our WNF through the removal of less thermally stable extractives, alkali-sensitive lignin, and hemicelluloses.

\section{Redispersion of wood and reference nanofibers}

The abilities of dried nanofibers to be redispersed and to maintain their original properties without irreversible aggregation were studied. First, nanopapers were fabricated in water or ethanol and left to dry freely. The freely dried nanopapers (Fig. 7a, c, d) showed characteristic nanocellulosic features, as they were compact, stiff, and tended to wrinkle strongly in the course of drying. This, however, did not occur with the WNF nanopapers dried in ethanol (Fig. 7b), which were actually the most brittle of the four samples. The WNF had a differing structure when ethanol was applied as a drying medium, and the nanofibers formed a less-compacted and loosely connected porous network structure (Visanko et al. 2014b). The stiffness and reduced polarity of the WNF were thought to have contributed to the ready nanopaper formation. Also, a lighter color was observed between the WNF nanopapers (Fig. 7a, b), which originated from the structural differences between the specimens. With the reference nanofibers, no clear structural differences between the nanopapers (Fig. 7 c, d) were noted, although the nanopaper fabricated in ethanol was hazier, which accounted for the increased nanoporosity of the nanopaper (Sehaqui et al. 2011).

After the dried nanopapers were redispersed into water and their viscosities at different rotational speeds were measured and compared to those of the original nanofibers. A $1.5-\mathrm{wt} \%$ consistency for the WNF was selected for the analysis, as this viscosity most resembled the reference nanofibers at a 0.3 -wt $\%$ consistency (see previous viscosity results) and was thus best for comparison. The solvent used in the fabrication of the reference nanopapers (water or

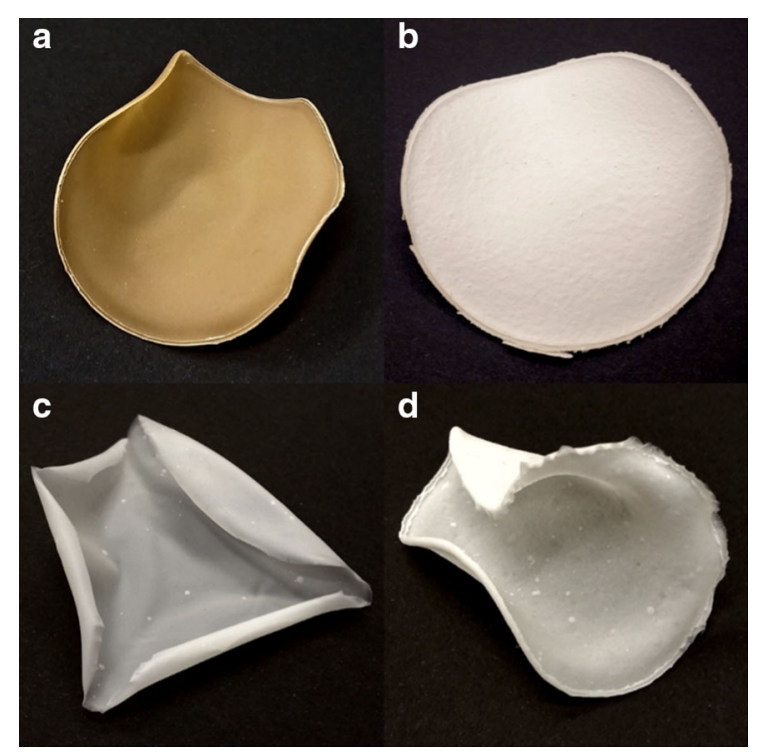

Fig. 7 Freely-dried nanopapers made from WNF and reference nanofibers. WNF dried in water (a), WNF dried in ethanol (b), and reference nanofibers dried in water (c) and ethanol (d) 
ethanol) did not impart the properties of the redispersed nanofibers, as those viscosities were similar (Fig. 8a). The viscosities of the redispersed reference nanofibers could be recovered by only 44-53\% (REF RW) and 45-55\% (REF RE) depending on the applied rotational speed of the spindle, when compared to the original reference nanofibers without redispersion (REF W). The WNF redispersed from the nanopaper dried in water (WNF RW) showed a similar behavior to the references, retaining viscosities around 45-63\% in comparison to the original WNF (WNF W). The WNF redispersed from the nanopaper dried in ethanol (WNF RE) maintained the highest recovery rate of all the specimens, having viscosities of $80-96 \%$ in contrast to the original WNF W. This exceptionally high recovery in viscosity was attained after the
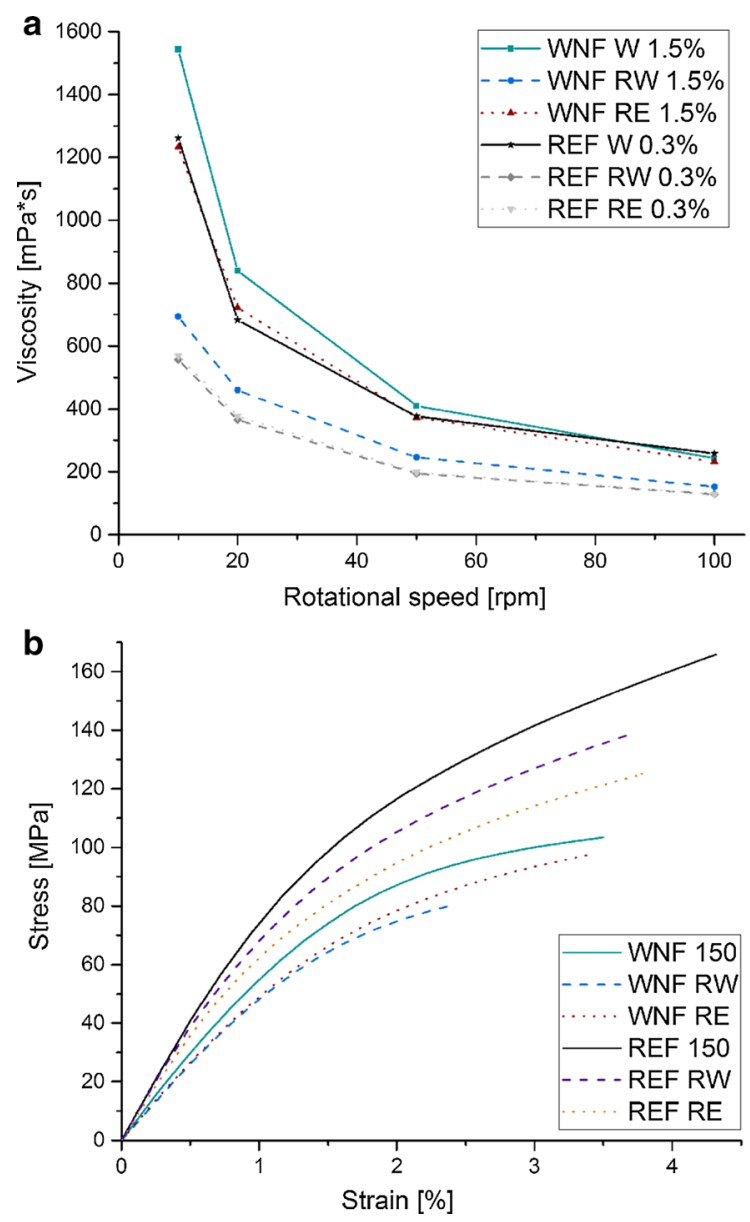

Fig. 8 Viscosity (a) and stress-strain curves (b) for both redispersed and original WNF and reference nanofiber samples successful individualization of the redispersed nanofibers into their original forms.

The strength of a nanopaper fabricated from nanosized components is susceptible to any irregularities or changes in the network structure. The formation of larger nanofiber bundles via aggregation decreases the contact points within the nanofiber network and can result in random weak points that are prone to breakage. This phenomenon was observed through the premature rupture of the nanopapers manufactured from redispersed nanofibers; also, decreases in the tensile strength, strain-at-break, and elastic moduli were recorded (Fig. 8b; see Table S2 for detailed values). The only exception was the nanopaper fabricated from the redispersed WNF from ethanol-dried nanopaper (WNF RE), which, with successful redispersion, had mechanical properties that were close to those of the original WNF (WNF 150 ), with only a minor decrease in stiffness and strength. The redispersed reference nanofibers showed a behavior opposite to the WNF specimens, as the redispersed nanopaper from the water-based nanopaper (REF RW) was able to revive with superior properties compared to that made after ethanol-based drying (REF RE). Together, the viscosity and tensile testing results strongly indicate, for the first time, that solely by capitalizing on WNF in their natural forms without any chemical modification, a novel yet simple approach to nanopaper fabrication resulted in successful redispersion and recovery without any significant losses of the original WNF properties.

\section{Conclusions}

The readily adaptable chemical-free processing of GWP to produce WNF, based on high-temperature disc nanogrinding, has been presented. The preservation of the lignin components in the WNF was found to result in unique advantageous characteristics of the nanofibers. The processing of the GWP was possible at an elevated consistency of above $4 \mathrm{wt} \%$, which can be considered auspicious for increasing the production capacity of the nanofibers. The WNF had a good compatibility with ethanol, and the reported viscosities of the different solutions were higher than those measured in water. Using ethanol as a drying medium for nanopapers fabricated from the WNF, it was possible to obtain the original properties when the nanofibers were 
redispersed back into water, which was not possible with the bleached-cellulose reference nanofibers. This characteristic makes the use of WNF compelling for largerscale applications, in whi8ch the dried nanofibers could be reused without a notable degradation of their properties, and their transportation cost could be significantly reduced by eliminating water. Water removal from the WNF was found to be highly suitable for this purpose, as only $3.5 \mathrm{~min}$ was required for nanopaper $\left(80 \mathrm{~g} / \mathrm{m}^{2}\right)$ fabrication, which could resolve many current problems relating to the difficulties of processing associated with nanofibers that are apt to bind to water. The WNF had hydrophobic surfaces, their thermal stability was not reduced after nanofiber preparation, and they were able to disperse into lesspolar solvents, all of which are advantageous for their suitability as reinforcing fillers in biocomposite manufacturing. We predict that the use of WNF with a high lignin content will become more frequent, and that they will be found to be appropriate for many new applications due to their unique properties and greatly reduced cost of production. The applicability of fabricated WNF can be further broadened by introducing new functionalities to the fiber surfaces, a topic on which we are aiming to publish in the near future.

Acknowledgments We thank Ilkka Miinalainen and Sami Saukko for their help with the TEM imaging. Marcin Selent, Tommi Kokkonen, and Jarno Karvonen are acknowledged for performing the XRD, TGA, and FiberLab analyses, respectively. The facilities at the Center of Microscopy and Nanotechnology at the University of Oulu were used in this research.

Open Access This article is distributed under the terms of the Creative Commons Attribution 4.0 International License (http:// creativecommons.org/licenses/by/4.0/), which permits unrestricted use, distribution, and reproduction in any medium, provided you give appropriate credit to the original author(s) and the source, provide a link to the Creative Commons license, and indicate if changes were made.

\section{References}

Abe K, Iwamoto S, Yano H (2007) Obtaining cellulose nanofibers with a uniform width of $15 \mathrm{~nm}$ from wood. Biomacromolecules 8:3276-3278. doi:10.1021/bm700624p

Arppe M (2001) Mechanical pulp—has it got a future or will it be discontinued? Int Pap 10:45-50

Bhatnagar A, Sain M (2005) Processing of cellulose nanofiberreinforced composites. J Reinf Plast Compos 24:12591268. doi: $10.1177 / 0731684405049864$
Butchosa N, Zhou Q (2014) Water redispersible cellulose nanofibrils adsorbed with carboxymethyl cellulose. Cellulose 21:4349-4358. doi:10.1007/s10570-014-0452-7

Chen W, Yu H, Liu Y et al (2011) Individualization of cellulose nanofibers from wood using high-intensity ultrasonication combined with chemical pretreatments. Carbohydr Polym 83:1804-1811. doi:10.1016/j.carbpol.2010.10.040

Clark GL, Terford HC (1955) Quantitative X-ray determination of amorphous phase in wood pulps as related to physical and chemical properties. Anal Chem 27:888-895. doi:10. 1021/ac60102a006

Donius AE, Liu A, Berglund LA, Wegst UGK (2014) Superior mechanical performance of highly porous, anisotropic nanocellulose-montmorillonite aerogels prepared by freeze casting. J Mech Behav Biomed Mater 37:88-99. doi:10.1016/j.jmbbm.2014.05.012

Eyholzer C, Bordeanu N, Lopez-Suevos F et al (2010) Preparation and characterization of water-redispersible nanofibrillated cellulose in powder form. Cellulose 17:19-30. doi:10.1007/s10570-009-9372-3

Fukuzumi H, Saito T, Iwata T et al (2009) Transparent and high gas barrier films of cellulose nanofibers prepared by TEMPOmediated oxidation. Biomacromolecules 10:162-165. doi:10. 1021/bm801065u

Gani A, Naruse I (2007) Effect of cellulose and lignin content on pyrolysis and combustion characteristics for several types of biomass. Renew Energy 32:649-661. doi:10.1016/j. renene.2006.02.017

Goring D (1963) Thermal softening of lignin, hemicellulose and cellulose. Pulp Pap Mag Can 64:517-527

Habibi Y (2014) Key advances in the chemical modification of nanocelluloses. Chem Soc Rev 43:1519-1542. doi:10. 1039/c3cs60204d

Herrick FW, Casebier RL, Hamilton JK, Sandberg KR (1983) Microfibrillated cellulose: morphology and accessibility. J Appl Polym Sci Appl Polym Symp 37:797-813

Herzele S, Veigel S, Liebner F et al (2016) Reinforcement of polycaprolactone with microfibrillated lignocellulose. Ind Crops Prod 93:302-308. doi:10.1016/j.indcrop.2015.12. 051

Hiasa S, Kumagai A, Endo T, Edashige Y (2016) Prevention of aggregation of pectin-containing cellulose nanofibers prepared from mandarin peel. J Fiber Sci Technol 72:17-26. doi:10.2115/fiberst.2016-0006

Hietala M, Sain S, Oksman K (2017) Highly redispersible sugar beet nanofibers as reinforcement in bionanocomposites. Cellulose. doi:10.1007/s10570-017-1245-6

Hoeger IC, Nair SS, Ragauskas AJ et al (2013) Mechanical deconstruction of lignocellulose cell walls and their enzymatic saccharification. Cellulose 20:807-818. doi:10. 1007/s10570-013-9867-9

Hu C, Zhao Y, Li K, et al (2015) Optimizing cellulose fibrillation for the production of cellulose nanofibrils by a disk grinder. Holzforschung 69:993-1000

Kim U-J, Eom SH, Wada M (2010) Thermal decomposition of native cellulose: influence on crystallite size. Polym Degrad Stab 95:778-781. doi:10.1016/j.polymdegradstab. 2010.02.009

Kisonen V, Prakobna K, Xu C et al (2015) Composite films of nanofibrillated cellulose and $\mathrm{O}$-acetyl galactoglucomannan (GGM) coated with succinic esters of GGM showing 
potential as barrier material in food packaging. J Mater Sci 50:3189-3199. doi:10.1007/s10853-015-8882-7

Korhonen JT, Hiekkataipale P, Malm J et al (2011) Inorganic hollow nanotube aerogels by atomic layer deposition onto native nanocellulose templates. ACS Nano 5:1967-1974. doi:10.1021/nn200108s

Kuutti L, Pajari H, Rovio S et al (2016) Chemical recovery in TEMPO oxidation. BioResources 11:6050-6061. doi:10. 15376/biores.11.3.6049-6061

Laitinen O, Hartmann R, Sirviö JA et al (2016) Alkyl aminated nanocelluloses in selective flotation of aluminium oxide and quartz. Chem Eng Sci 144:260-266. doi:10.1016/j.ces. 2016.01.052

Laurichesse S, Avérous L (2014) Chemical modification of lignins: towards biobased polymers. Prog Polym Sci 39:1266-1290. doi:10.1016/j.progpolymsci.2013.11.004

Lavoine N, Desloges I, Dufresne A, Bras J (2012) Microfibrillated cellulose-its barrier properties and applications in cellulosic materials: a review. Carbohydr Polym 90:735764. doi:10.1016/j.carbpol.2012.05.026

Li P, Sirviö JA, Haapala A, Liimatainen H (2017) Cellulose nanofibrils from nonderivatizing urea-based deep eutectic solvent pretreatments. ACS Appl Mater Interfaces. doi:10. 1021/acsami.6b13625

Liimatainen H, Visanko M, Sirviö JA et al (2012) Enhancement of the nanofibrillation of wood cellulose through sequential periodate-chlorite oxidation. Biomacromolecules 13:15921597. doi:10.1021/bm300319m

Liimatainen H, Sirviö J, Pajari H et al (2013a) Regeneration and recycling of aqueous periodate solution in dialdehyde cellulose production. J Wood Chem Technol 33:258-266. doi:10.1080/02773813.2013.783076

Liimatainen H, Visanko M, Sirviö J et al (2013b) Sulfonated cellulose nanofibrils obtained from wood pulp through regioselective oxidative bisulfite pre-treatment. Cellulose 20:741-749. doi:10.1007/s10570-013-9865-y

Lowys M-P, Desbrières J, Rinaudo M (2001) Rheological characterization of cellulosic microfibril suspensions. Role of polymeric additives. Food Hydrocoll 15:25-32. doi:10. 1016/S0268-005X(00)00046-1

McDonald D, Miles K, Amiri R (2004) The nature of the mechanical pulping process. Pulp Pap Can 105:27-32

Morales LO, Iakovlev M, Martin-Sampedro R et al (2014) Effects of residual lignin and heteropolysaccharides on the bioconversion of softwood lignocellulose nanofibrils obtained by SO2-ethanol-water fractionation. Bioresour Technol 161:55-62. doi:10.1016/j.biortech.2014.03.025

Morales-Narváez E, Golmohammadi H, Naghdi T et al (2015) Nanopaper as an optical sensing platform. ACS Nano 9:7296-7305. doi:10.1021/acsnano.5b03097

Morán JI, Alvarez VA, Cyras VP, Vázquez A (2008) Extraction of cellulose and preparation of nanocellulose from sisal fibers. Cellulose 15:149-159. doi:10.1007/s10570-0079145-9

Nair SS, Yan N (2015a) Bark derived submicron-sized and nano-sized cellulose fibers: from industrial waste to high performance materials. Carbohydr Polym 134:258-266. doi:10.1016/j.carbpol.2015.07.080

Nair SS, Yan N (2015b) Effect of high residual lignin on the thermal stability of nanofibrils and its enhanced mechanical performance in aqueous environments. Cellulose 22:3137-3150. doi:10.1007/s10570-015-0737-5

Nair SS, Zhu JY, Deng Y, Ragauskas AJ (2014) Characterization of cellulose nanofibrillation by micro grinding. J Nanoparticle Res 16:2349. doi:10.1007/s11051-0142349-7

Newman RH (2004) Carbon-13 NMR evidence for cocrystallization of cellulose as a mechanism for hornification of bleached kraft pulp. Cellulose 11:45-52. doi:10.1023/B: CELL.0000014768.28924.0c

Nogi M, Iwamoto S, Nakagaito AN, Yano H (2009) Optically transparent nanofiber paper. Adv Mater 21:1595-1598. doi:10.1002/adma.200803174

Ojala J, Sirviö JA, Liimatainen H (2016) Nanoparticle emulsifiers based on bifunctionalized cellulose nanocrystals as marine diesel oil-water emulsion stabilizers. Chem Eng J 288:312-320. doi:10.1016/j.cej.2015.10.113

Oksman K, Aitomäki Y, Mathew AP et al (2016) Review of the recent developments in cellulose nanocomposite processing. Compos Part Appl Sci Manuf 83:2-18. doi:10.1016/j. compositesa.2015.10.041

Poletto M, Zattera AJ, Forte MMC, Santana RMC (2012) Thermal decomposition of wood: influence of wood components and cellulose crystallite size. Bioresour Technol 109:148-153. doi:10.1016/j.biortech.2011.11.122

Quiévy N, Jacquet N, Sclavons M et al (2010) Influence of homogenization and drying on the thermal stability of microfibrillated cellulose. Polym Degrad Stab 95:306-314. doi:10.1016/j.polymdegradstab.2009.11.020

Rojo E, Peresin MS, Sampson WW et al (2015) Comprehensive elucidation of the effect of residual lignin on the physical, barrier, mechanical and surface properties of nanocellulose films. Green Chem 17:1853-1866. doi:10.1039/C4GC02 398F

Saito T, Kimura S, Nishiyama Y, Isogai A (2007) Cellulose nanofibers prepared by TEMPO-mediated oxidation of native cellulose. Biomacromolecules 8:2485-2491. doi:10. 1021/bm0703970

Segal L, Creely JJ, Martin AE, Conrad CM (1959) An empirical method for estimating the degree of crystallinity of native cellulose using the X-ray diffractometer. Text Res J 29:786-794. doi:10.1177/004051755902901003

Sehaqui H, Liu A, Zhou Q, Berglund LA (2010) Fast preparation procedure for large, flat cellulose and cellulose/inorganic nanopaper structures. Biomacromolecules 11:2195-2198. doi:10.1021/bm100490s

Sehaqui H, Zhou Q, Ikkala O, Berglund LA (2011) Strong and tough cellulose nanopaper with high specific surface area and porosity. Biomacromolecules 12:3638-3644. doi:10. 1021/bm2008907

Sehaqui H, Ezekiel Mushi N, Morimune S et al (2012) Cellulose nanofiber orientation in nanopaper and nanocomposites by cold drawing. ACS Appl Mater Interfaces 4:1043-1049. doi:10.1021/am2016766

Selkälä T, Sirviö JA, Lorite GS, Liimatainen H (2016) Anionically stabilized cellulose nanofibrils through succinylation pretreatment in urea-lithium chloride deep eutectic solvent. Chemsuschem 9:3074-3083. doi:10.1002/cssc.201600903

Sirviö JA, Anttila A-K, Pirttilä AM et al (2014a) Cationic wood cellulose films with high strength and bacterial anti- 
adhesive properties. Cellulose 21:3573-3583. doi:10.1007/ s10570-014-0351-y

Sirviö JA, Kolehmainen A, Visanko M et al (2014b) Strong, self-standing oxygen barrier films from nanocelluloses modified with regioselective oxidative treatments. ACS Appl Mater Interfaces 6:14384-14390. doi:10.1021/ am503659j

Sirviö JA, Hasa T, Ahola J et al (2015) Phosphonated nanocelluloses from sequential oxidative-reductive treatment-Physicochemical characteristics and thermal properties. Carbohydr Polym 133:524-532. doi:10.1016/j. carbpol.2015.06.090

Sixta H (ed) (2006) Handbook of pulp. Wiley, Weinheim

Spence KL, Venditti RA, Rojas OJ et al (2010) The effect of chemical composition on microfibrillar cellulose films from wood pulps: water interactions and physical properties for packaging applications. Cellulose 17:835-848. doi:10. 1007/s10570-010-9424-8

Tarrés Q, Espinosa E, Domínguez-Robles J et al (2017) The suitability of banana leaf residue as raw material for the production of high lignin content micro/nano fibers: from residue to value-added products. Ind Crops Prod 99:27-33. doi:10.1016/j.indcrop.2017.01.021

Valadez-Gonzalez A, Cervantes-Uc JM, Olayo R, Herrera-Franco PJ (1999) Effect of fiber surface treatment on the fiber- matrix bond strength of natural fiber reinforced composites. Compos Part B 30:309-320. doi:10.1016/S1359-8368(98) 00054-7

Visanko M, Liimatainen H, Sirviö JA et al (2014a) Amphiphilic cellulose nanocrystals from acid-free oxidative treatment: physicochemical characteristics and use as an oil-water stabilizer. Biomacromolecules 15:2769-2775. doi:10. 1021/bm500628g

Visanko M, Liimatainen H, Sirviö JA et al (2014b) Porous thin film barrier layers from 2,3-dicarboxylic acid cellulose nanofibrils for membrane structures. Carbohydr Polym 102:584-589. doi:10.1016/j.carbpol.2013.12.006

Winter A, Andorfer L, Herzele S et al (2017) Reduced polarity and improved dispersion of microfibrillated cellulose in poly(lactic-acid) provided by residual lignin and hemicellulose. J Mater Sci 52:60-72. doi:10.1007/s10853-0160439-X

Yang H, Yan R, Chen H et al (2007) Characteristics of hemicellulose, cellulose and lignin pyrolysis. Fuel 86:17811788. doi:10.1016/j.fuel.2006.12.013

Zhang S-Y, Fei B-H, Yu Y et al (2013) Effect of the amount of lignin on tensile properties of single wood fibers. For Sci Pract 15:56-60. doi:10.1007/s11632-013-0106-0 